This item was submitted to Loughborough's Research Repository by the author.

Items in Figshare are protected by copyright, with all rights reserved, unless otherwise indicated.

\title{
Silicon potentials investigated using density functional theory fitted neural networks
}

PLEASE CITE THE PUBLISHED VERSION

http://dx.doi.org/10.1088/0953-8984/20/28/285219

PUBLISHER

(c) IOP Publishing Ltd

VERSION

SMUR (Submitted Manuscript Under Review)

LICENCE

CC BY-NC-ND 4.0

\section{REPOSITORY RECORD}

Sanville, E., Ajeevsing Bholoa, Roger Smith, and Steven D. Kenny. 2019. "Silicon Potentials Investigated Using Density Functional Theory Fitted Neural Networks”. figshare. https://hdl.handle.net/2134/15407. 
This item was submitted to Loughborough's Institutional Repository (https://dspace.lboro.ac.uk/) by the author and is made available under the following Creative Commons Licence conditions.

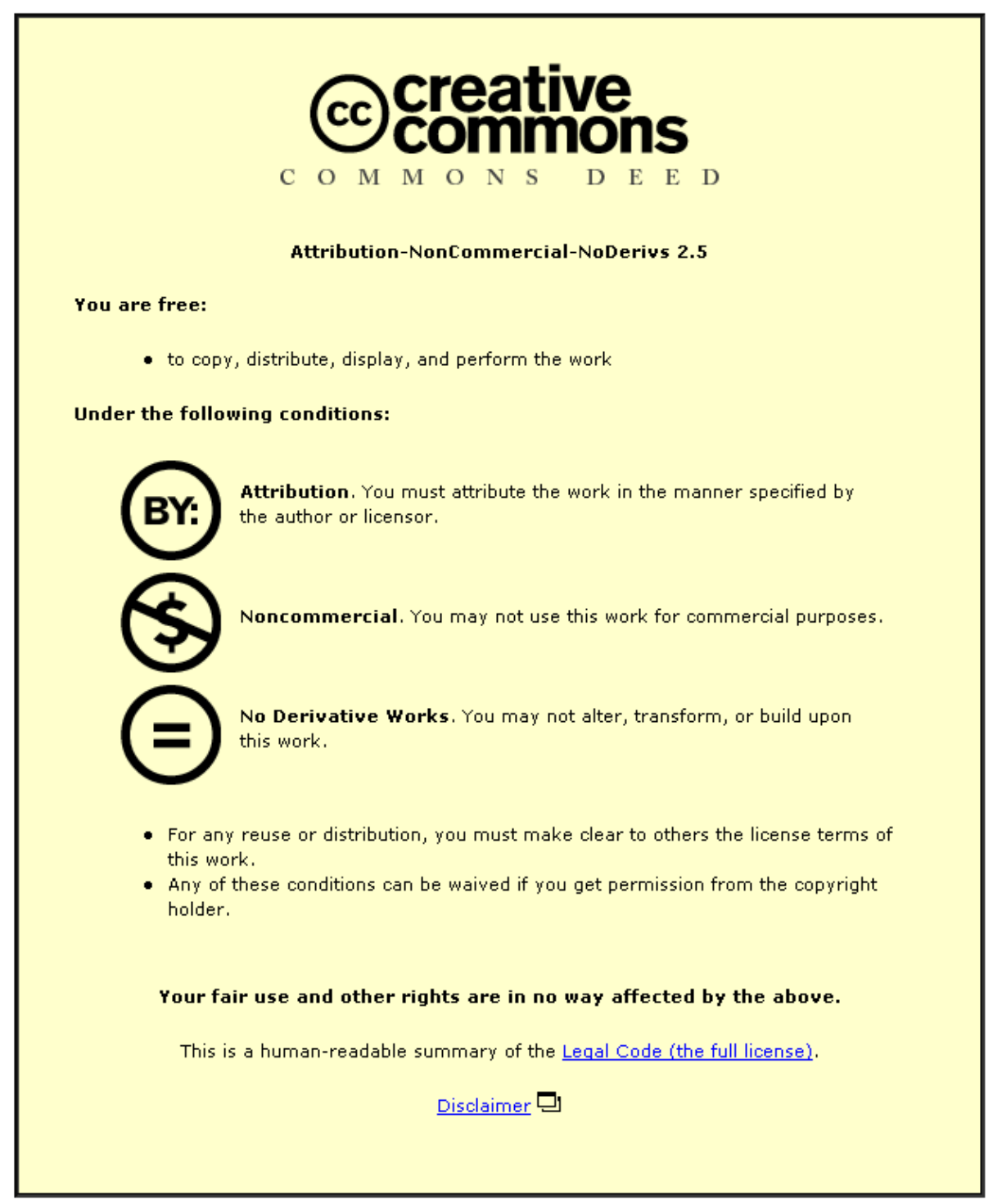

For the full text of this licence, please go to: http://creativecommons.org/licenses/by-nc-nd/2.5/ 


\title{
Silicon Potentials using Density Functional Theory-Fitted Neural Networks
}

\author{
E. Sanville, A. Bholoa, R. Smith, and S. D. Kenny \\ Department of Mathematical Sciences, Loughborough University, \\ Loughborough, United Kingdom LE11 3TU
}

(Dated: November 21, 2007)

\begin{abstract}
We present a method for fitting neural networks to geometric and energetic datasets. We then apply this method by fitting a neural network to a set of data generated using the local density approximation for systems composed entirely of silicon. In order to generate atomic potential energy data, we use the Bader analysis scheme to partition the total system energy among the constituent atoms. We then demonstrate the transferability of the neural network potential by fitting to various bulk, surface, and cluster systems.
\end{abstract}




\section{INTRODUCTION}

Today, there exist many schemes for the development of empirical potentials for application in molecular dynamics research. These schemes range from simple two-body potentials such as the Lennard-Jones Potential, through potential functions of intermediate complexity such as the glue model [1], the embedded atom method [2, 3], and the Finnis-Sinclair Potentials [4], to angular dependent forms including the Stillinger-Weber [5], and Tersoff [6] potentials. Potentials [1-4] involve both a sum of two-body interaction potentials, and a potential term which is a sum of a function of the generalised coordination of each atom. The coordination potential terms are formulated in such a way as to model changes in interatomic bond strength as a result of crowding and steric hindrance among neighboring atoms. While not of great importance in the modelling of noble gases, these terms are more significant in the modelling of metallic materials, especially those with increasing covalent bonding character. The Stillinger-Weber potential replaces the coordination terms of potentials [1-4] with a three-body angular term that introduces an energetic penalty which is a function of angular deviations from an angle of known stability. This angular potential energy term attempts to model atomic orbital hybridization, in addition to atomic crowding and changes in coordination. The Tersoff potential arguably improves on this concept by replacing the angular potential terms with a set of attractive terms for each interatomic interaction that are proportional to the bond order of each interaction. This bond order is in turn defined as a function of a coordination number corresponding to the bond $i j$. This bond coordination number is formulated using the bond angles and distances involving bonds $i j$ and all $i k$ $(k \neq j)$. While potentials $[5,6]$ have found some success in describing certain aspects of semiconductor systems, they still demonstrate serious transferability problems.

Meanwhile, cheaper availablity of more and more powerful computers has led to broader applicability of $a b$ initio electronic structure methods in recent years. In particular, density functional theory has experienced a boost of popularity, for physical, chemical, and materials science applications. Two decades ago, molecular dynamics simulations in which ions or nuclei moved classically in a potential field calculated by using ab initio methods were pioneered by Car and Parrinello[7]. These calculations bypassed the issue of empirical potential development entirely, avoiding many of the problems inherent in these potentials. This approach effectively uses the Born-Oppenheimer approximation to separate the dynam- 
ics simulation into two parts: the calculation of forces on the ions by differentiation of the electronic structure energy, and the movement of the atomic coordinates according to classical physics using a numerical integration algorithm [8]. The vast majority of computation time corresponds to the calculation of the forces on the nuclei. MD simulations can thus, in principle, arbitrarily approach $a b$ initio levels of accuracy without the vast computational expenses associated with such methods, by more accurately fitting the ab initio potential energy surfaces to more complex parametrised models, and replacing the differentiation of the electronic structure energy by the evaluation of the energy from these models.

Neural networks have been used successfully to model various complex systems[9-12], without the formation of an explicit model or parametrised formula. Instead, the artificial neural network is composed of a set of interconnected nodes or neurons, which individually take a set of input values and produce an output value based on a simple formula that takes a small number of parameters. In a feed-forward network, the entire neural network is composed of a number of layers of nodes. The first layer is composed of input nodes, which produce the independent variables as outputs. Each node of the second layer maintains a connection to each of the input nodes with a specific weight, $w_{Q P}^{A}$. The inputs to each of the second layer nodes are then simply the product of the weight with the output from each of the input nodes. This sum is then passed as the parameter of an activation function $\phi$. The output from each node is then evaluated as the sum of this activation function and the bias of the node $b_{Q}^{A}$. A similar process is used for the third layer of nodes, using the weights $w_{R Q}^{B}$, and the outputs from the second layer, and so on. The final layer is called the output layer, and the outputs from this layer are the predicted values of the dependent variables of the model.

\section{NEURAL NETWORK}

Previously, we have reported a neural network potential, similar in design to the present algorithm, except with fewer input variables, that was fit to a smaller subset of tight-binding energetics data [13]. The present algorithm improves on this design by fitting a larger subset of $a b$ initio data, using some additional input variables.

Recently, an algorithm for generating an interatomic potential for silicon using a neural network was reported [14]. This neural network used a set of symmetry functions to char- 


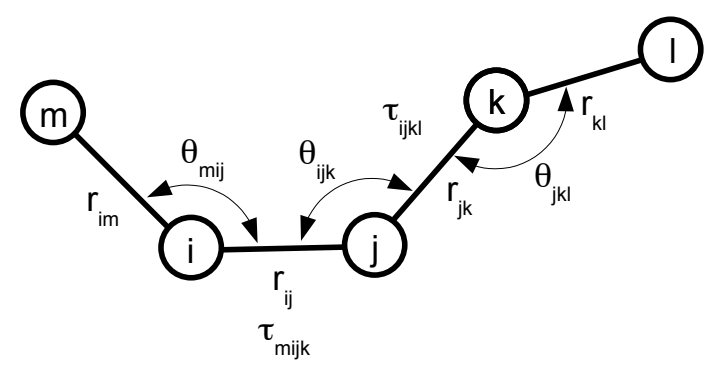

FIG. 1: Input variables.

acterise the environment of each atom, and used these symmetry functions as input to the neural network. An advantage of this network design was that the number of input variables for each atom was constant, and independent of the geometry of the neighborhood of the atom.

In this paper, we present a different method for generating a many-body interatomic potential from ab initio data, using a feed-forward network topology. In constrast to previously reported network topologies, this network has a variable number of input variables for each atom in the system. Additionally, the complete geometric environment of each atom is explicitly taken into account, including local bond lengths, angles, and torsion angles. Input to the neural network is composed of a series of vectors, each corresponding to a five-atom chain found within the system. Each vector completely describes the geometry of the five atom chain using the following set of 9 input variables: $r_{i j}, r_{j k}, r_{k l}, r_{i m}, \cos \theta_{i j k}, \cos \theta_{j k l}$, $\cos \theta_{m i j}, \tau_{i j k l}$, and $\tau_{m i j k}$, (see Figure 1). In addition to these geometric input variables, the following additional variables are used:

$$
\begin{gathered}
N_{i}=\sum_{j} S_{i j} \\
N_{j}=\sum_{k \neq i} S_{j k} \\
N_{m}=\sum_{k \neq i} S_{m k} \\
N_{\text {inputs }}=\sum_{j \neq i} S_{i j}\left(1+\sum_{k \neq i, j} S_{j k}\left(1+\sum_{l \neq i, j, k} S_{k l}\left(1+\sum_{m \neq i, j, k, l} S_{i m}\right)\right)\right)
\end{gathered}
$$

where $i, j, k, l$, and $m$ are the indices of atoms, and $S_{i j}$ is the bond screening factor between atoms $i$ and $j$ (described below). The sums with index $j$ are over the neighbor list associated with atom $i$, while the sums with index $k$ are over the neighbor list associated 
with atom $j$, and so on. The neighbor list was generated with a cutoff distance of $8 \AA$. The two-center bond screening factors $S_{i j}$ were calculated as the product of all three-center bond screening factors:

$$
S_{i j}=f\left(r_{i j}\right) \prod_{k \neq i, j} S_{i k j}
$$

where the product is over all atoms $k$ which are found on the neighbor lists of both atom $i$ and atom $j$, and $f\left(r_{i j}\right)$ is a distance screening function, (distances measured in Ångstroms):

$$
f\left(r_{i j}\right)= \begin{cases}1 & r_{i j}<6.5 \\ \frac{1}{2}\left[\cos \left(\frac{\pi}{1.5}\left(r_{i j}-6.5\right)\right)+1\right] & 6.5<r_{i j}<8.0 \\ 0 & r_{i j}>8.0\end{cases}
$$

The three-center bond screening factors $S_{i k j}$ were calculated using the scheme of Baskes [15]. A total screening factor $S^{(n)}$ is calculated for each input vector $\mathbf{x}^{(n)}$ :

$$
S^{(n)}=S_{m i} S_{i j} S_{j k} S_{k l}
$$

All possible five-atom chains involving atom $i$ with $S^{(n)} \neq 0$ are used as input for the neural network. The output of the neural network is the predicted energy of atom $i$, (Figure 2).

From each input vector $\mathbf{x}^{(n)}$, the outputs from the first hidden layer $\mathbf{y}^{A(n)}$ are calculated. In this model, there is always the same number of nodes in the second hidden layer as there are for one of the first hidden layers. The inputs to the second hidden layer are then calculated as the linear combination of the corresponding outputs from all of the first hidden layers from each of the input vectors. The weight of each first hidden layer $\mathbf{y}^{A(n)}$ is the total screening factor $S^{(n)}$. All inputs and outputs are linearly scaled such that the entire dataset occurs in the range $0.1<x<0.9$.

The set of geometry and energy data was divided up on a per-atom basis, in which $60 \%$ of the atoms were assigned to the training set, $20 \%$ were assigned to the validation set, and $20 \%$ were assigned to the test set. All weights and biases were optimised to minimise the sum of the squares of the errors within the training set, while not increasing the error of the validation set. The neural network was composed of 13 nodes in each of the second and third hidden layers. 


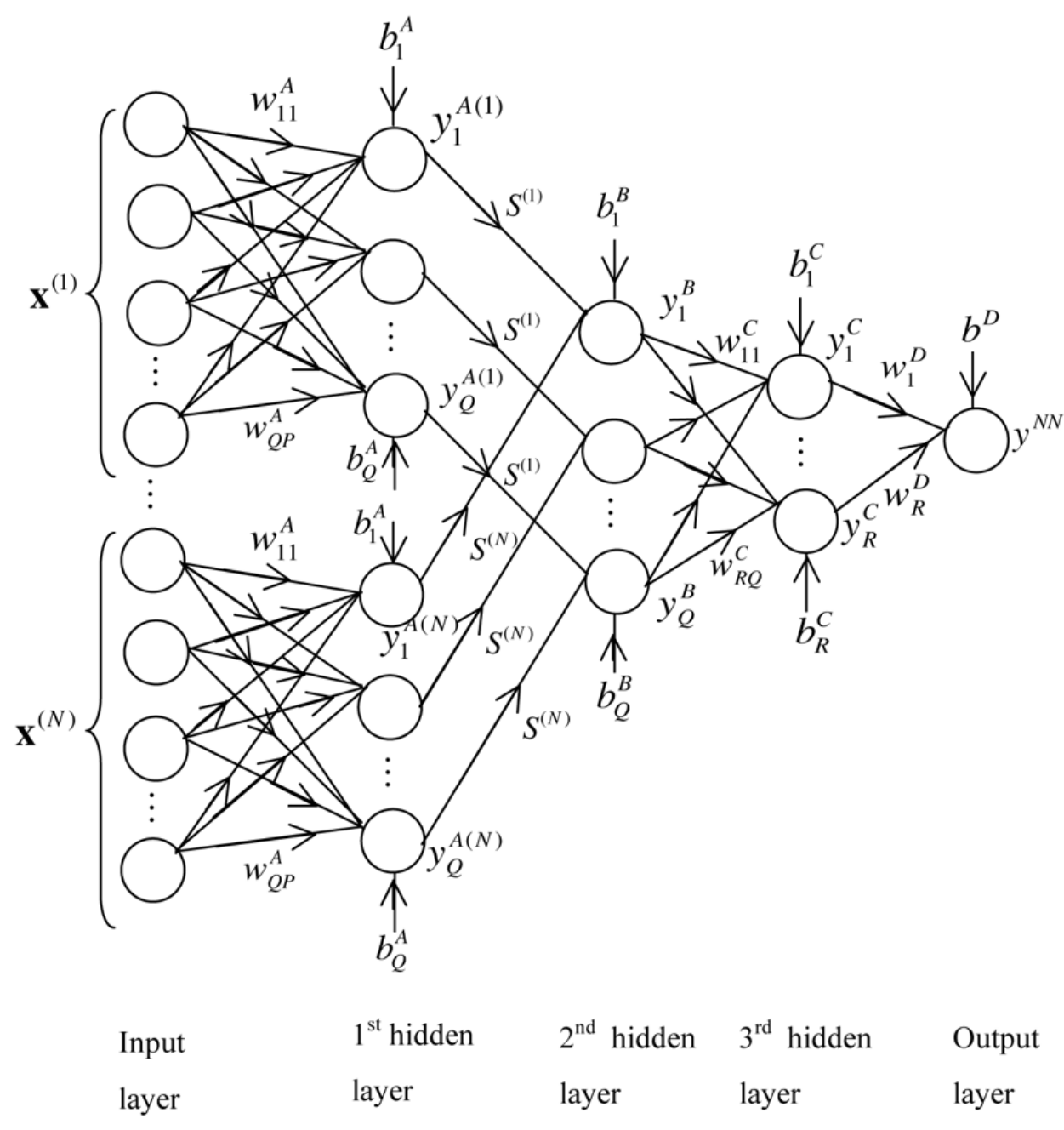

FIG. 2: Neural Nework model.

\section{BADER ANALYSIS}

One of the challenges of using $a b$ initio data to fit empirical potentials is the division of the total system energy among the atoms composing the system. Here, the Atoms in Molecules approach is used to assign regions of space to each atom in the system [16]. In this article, we use the Bader DFT energy allocation method previously reported by us [17].

\section{RESULTS}

The results of the neural network fitting procedure are shown in Figures 3-9. The dataset contained 260 single point symmetric clusters, for $\mathrm{Si}_{2-4}$. For the $\mathrm{Si}_{4}$ clusters, both squares and tetrahedra were used in the dataset, (Figure 3). Additionally, 225 asymmetric $\mathrm{Si}_{3}$ clus- 
ters were used, with interatomic angles varying between $0-180^{\circ}$, and interatomic distances ranging between $1-8 \AA$, (Figure 4). A set of 330 distorted bulk diamond systems were used, with varying hydrostatic compression strains, $C^{\prime}$ distortion strains, and $C_{44}$ distortion strains. The predicted and $a b$ initio energies of these bulk diamond systems are shown in Figure 5. Both hexagonal and tetrahedral interstitial systems were present within the dataset, including 32 systems composed of a $2 \times 2 \times 2$ unit cell with various cell parameters. The resulting stress-strain curves, and plots showing the predicted and actual energies of the lattice silicon atoms with respect to their distances from the interstitial are shown in Figure 6. Relaxed $2 \times 1$ and $2 \times 2$ reconstructed (001) model systems were also included, as slabs of 14-atom thickness. A set of $202 \times 1$ reconstructed (001) surface slabs with varying dimer tilt angles was also included in the dataset, (Figure 7). The tilt angles ranged between $0-19^{\circ}$, with $19^{\circ}$ being the energy minimum. Finally, silicon $\beta$-tin, BCT5, and ST12 systems were included with various hydrostatic compression strains, and $a / c$ ratios were included in the dataset, along with BC8 systems with both hydrostatic compression strains, and $C^{\prime}$ strains, (Figures 8-9). The optimised neural network weights and biases are given in the Appendix.

\section{CONCLUSIONS}

It has been demonstrated that neural networks can be used to fit the potential energy surfaces associated with a diverse set of $a b$ initio data for silicon. Small clusters, distorted Si-diamond, $\beta$-tin, BCT5, BC8, and ST12 unit cells, interstitial defects, and reconstructed surfaces have been fit using the screened neural network scheme described in this paper. The energetics of silicon clusters, bulk, and diamond (001) surfaces have been successfully modeled simultaneously with the same novel potential, based upon the results of ab-initio density-functional theory calculations. Additionally, the energy dependence upon the silicon diamond 001 surface dimer tilt angles has been fit successfully. It has been demonstrated that the neural network design is capable of fitting not only the total system energies, but also the individual atomic energies from the energy partitioning scheme. The neural network design allows the use of a variable number of inputs, by using a screening factor to combine the first hidden layer nodes for each of a set of input vectors. Each input vector fully describes

the geometry of a five-atom chain containing the atom of interest. The first hidden layer nodes for each input vector are combined using a screening factor which is the product of 

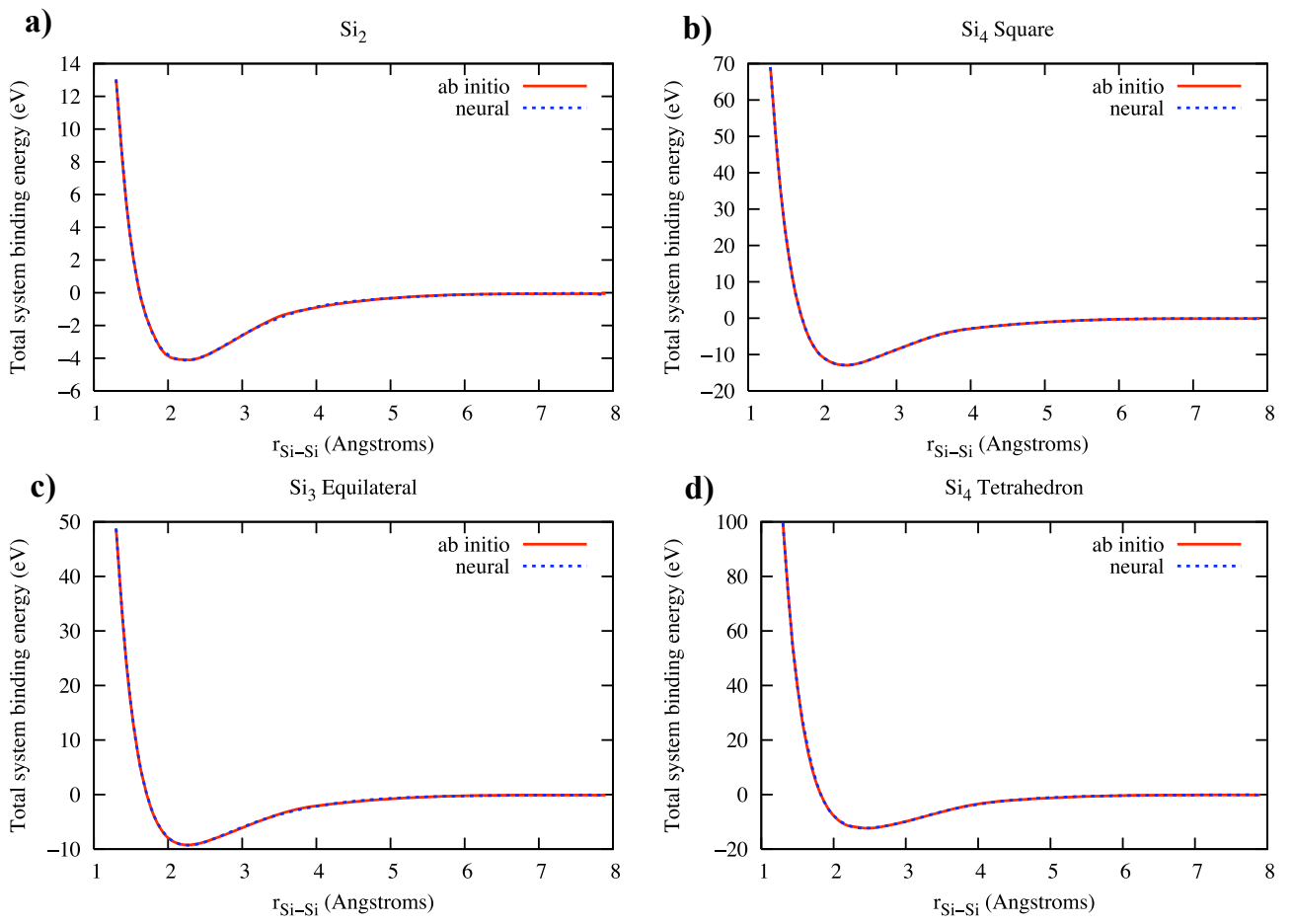

FIG. 3: Neural network fits for symmetric clusters: (a) $\mathrm{Si}_{2}$ (b) $\mathrm{Si}_{4}$ square (c) $\mathrm{Si}_{3}$ equilateral triangle (d) $\mathrm{Si}_{4}$ tetrahedron. Energetics for the clusters are compared for $r_{S i-S i}$ between 1.2 and $8.0 \AA$.

the screening factors associated with each of the four interatomic bonds within the five-atom chain. The interatomic screening factors are products of a distance screening function, and an atom screening function previously reported by Baskes, et. al. The distance screening function ranges from 0 to 1 , and has a smooth first derivative. Due to the fact that the geometry is characterised in terms of only interatomic distances, angles, and torsion angles, the input representation is independent of the axes used to describe the atomic coordinates, and is invariant to rotation of the system. Due to the nature of the screening factors, the input vectors are continuous and smooth with respect to movement of atoms within the system, which is a necessary condition for obtaining smooth potential energy curves from the neural network's output.

Two limitations of the neural network design are the large number of input parameters required for some systems, (particularly the BC8 systems), and a lack of the ability to 
a)

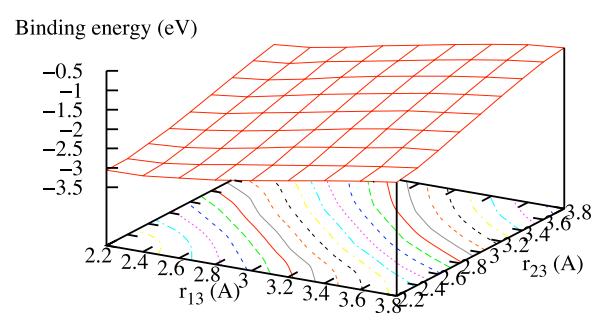

c)

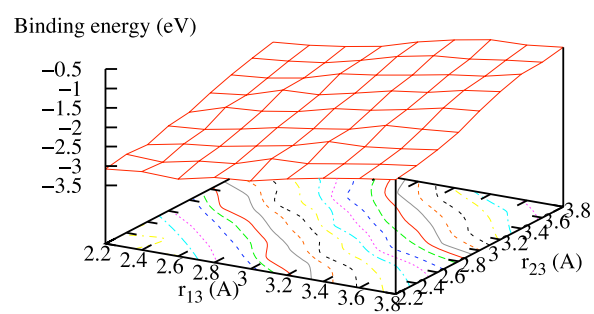

b)

$\mathrm{Si}_{3} \theta_{213}=120^{\circ}$ ab initio

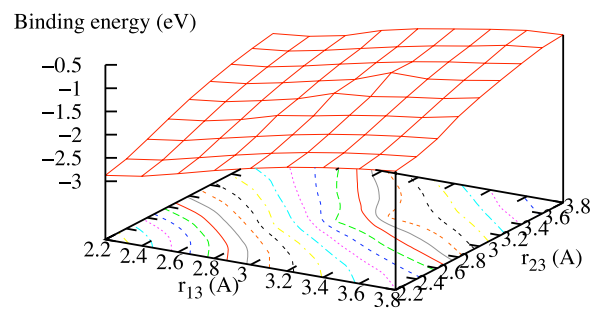

d)

$\mathrm{Si}_{3} \theta_{213}=120^{\circ}$ neural

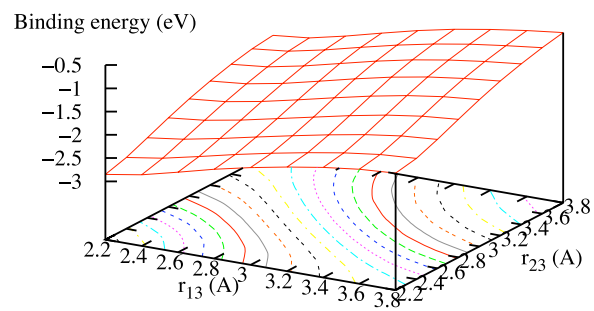

FIG. 4: Neural network fits on potential energy surfaces for asymmetric $S i_{3}$ clusters: (a) and (b) show $a b$ initio calculations for the potential energy surface with one angle of the triangle held constant, $\left(60^{\circ}\right.$ and $120^{\circ}$, respectively). (c) and (d) show the same potential energy surfaces as predicted by the neural network.

extrapolate to other system geometries outside of the training set. The major reason for the large number of input parameters is the redundancy of the input data, as each interatomic distance, angle and torsion can often be present several times in the input data. This is because each bond, angle and torsion angle can be part of several different five-atom chains. The lack of extrapolation ability is an inherent limitation of any neural network, and can be remedied by taking systematically larger sets of data into the training set.

Despite these limitations, the neural network potentials could be useful to obtain $a b$ initio accuracy at a small fraction of the computational cost. Forces can easily be calculated by differentiating the total system energy with respect to the coordinates of the atoms in the system. This allows the possibility of molecular dynamics simulations based upon the 
a)

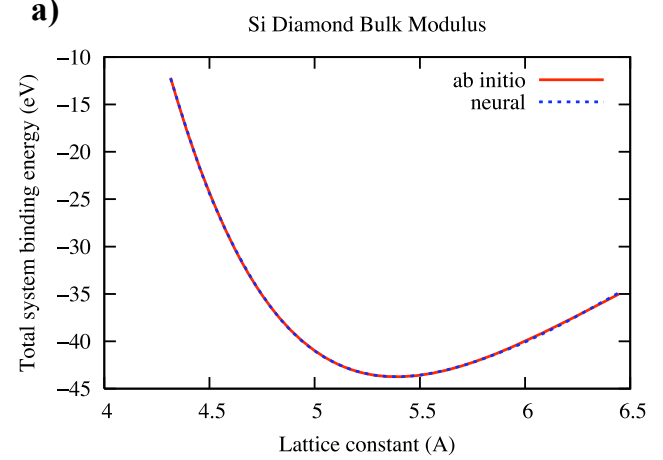

c)

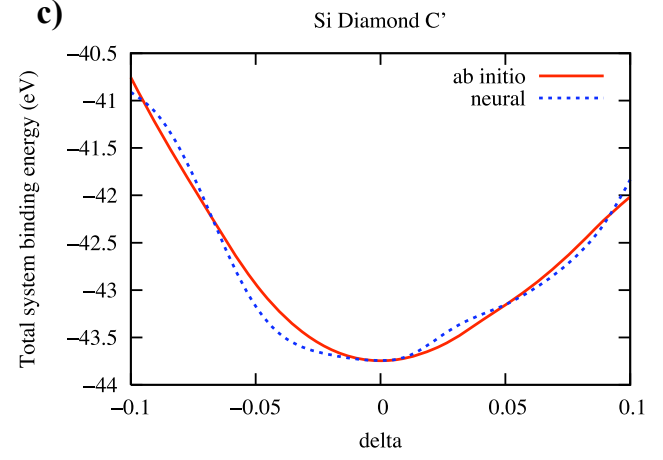

b)

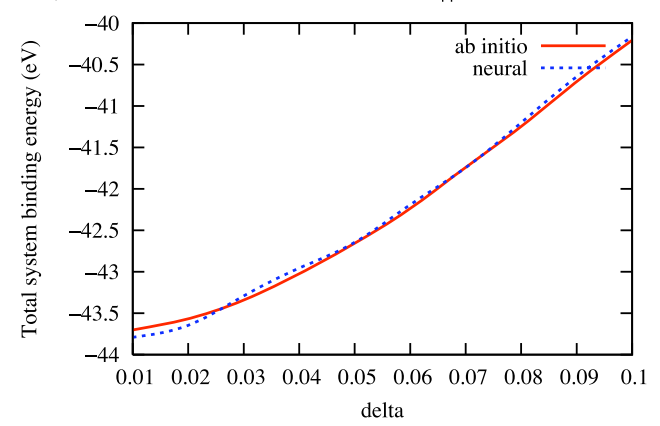

FIG. 5: Neural network fits for silicon diamond lattice distortions: (a) hydrostatic (bulk modulus), (b) $C_{44}$, and (c) $C^{\prime}$.

neural network potential. Additionally, the network can be generalised to multi-component systems. This would be accomplished most easily by adding a set of five additional input variables, which represent the element of each atom in the chain, and by fitting interatomic potentials between multiple different elements. 
a) Diamond $2 \times 2 \times 2$ Hexagonal Interstitial Atomic Energy by Distance

b) Diamond $2 \times 2 \times 2$ Tetrahedral Interstitial Atomic Energy by Distance
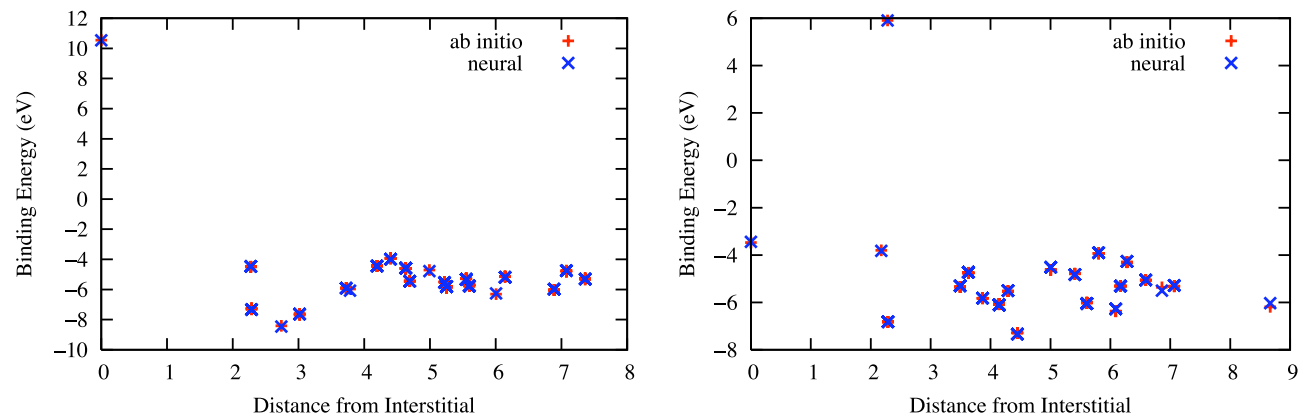

c) Si Diamond $2 \times 2 \times 2$ Hexagonal Interstitial Bulk Modulus
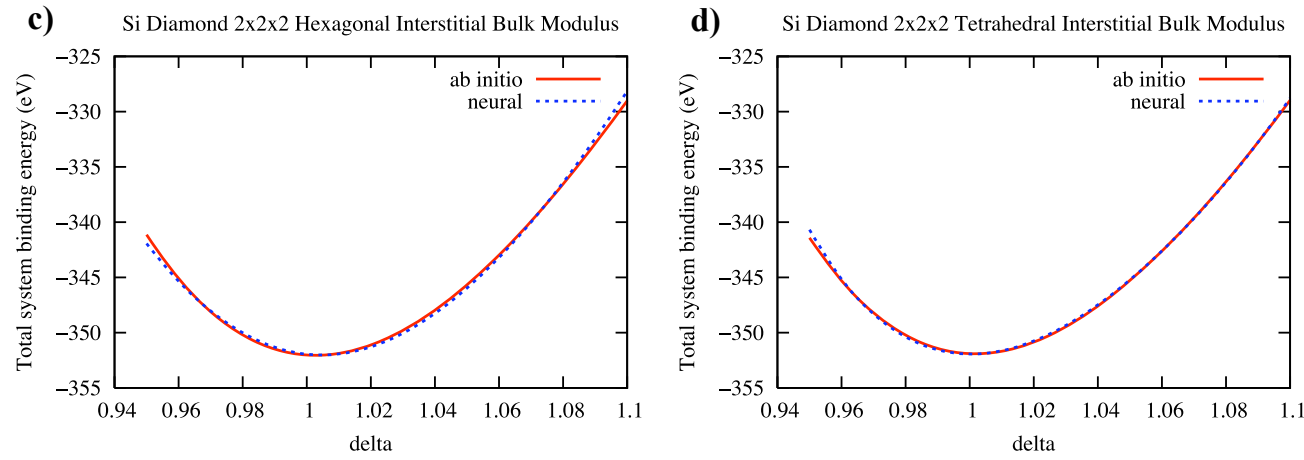

FIG. 6: Neural network fits for silicon diamond lattice interstitials. (a) and (b) Energies of individual lattice atoms are plotted with respect to their distance from a hexagonal and tetrahedral interstitial, respectively. (c) and (d) The $2 \times 2 \times 2$ unit cells undergo hydrostatic compression. 
Diamond 001 surface $2 \times 1 \times 3$ dimer tilted reconstruction
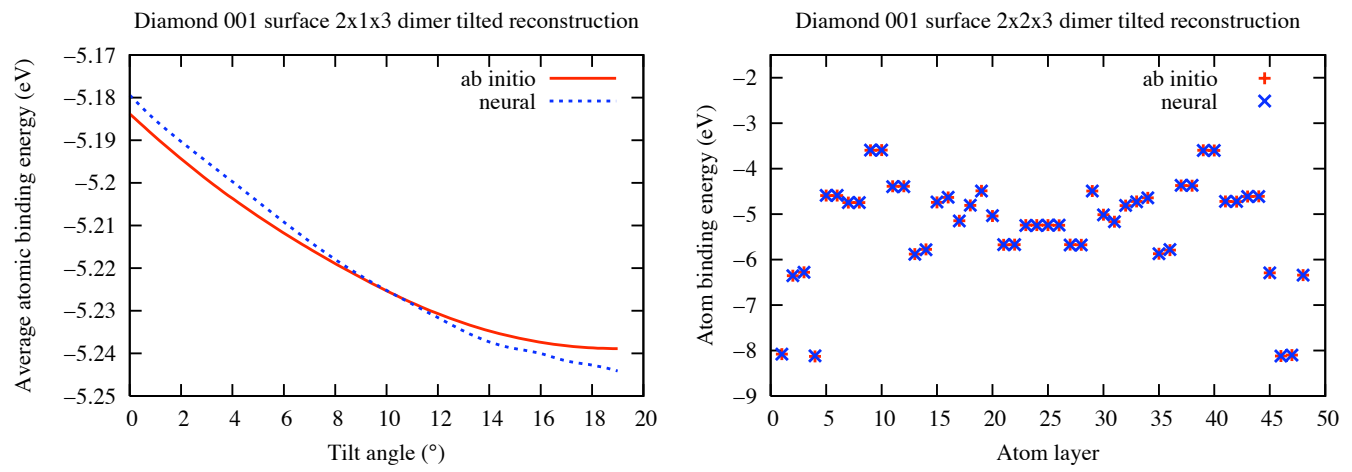

Diamond 001 surface $2 \times 1 \times 3$ dimer tilted reconstruction

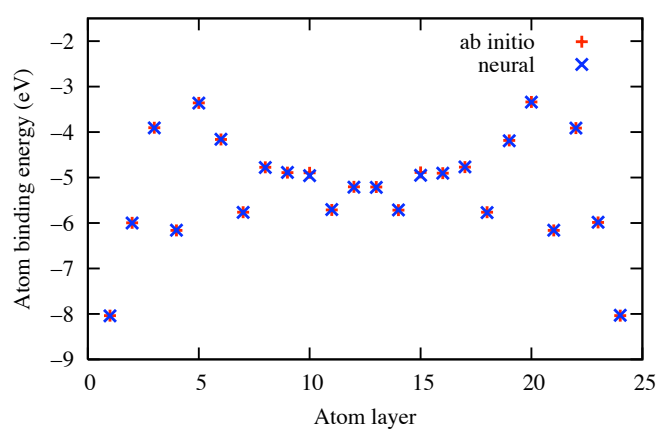

FIG. 7: Neural network fits for diamond (001) $2 \times 1$ and $2 \times 2$ reconstructed surfaces. (a) Energy is plotted against dimer tilt angle, with the tilt angle varying from 0-19 ${ }^{\circ}$. (b) and (c) Energies of individual atoms are plotted with respect to their depth into the slab, for the $2 \times 2$ and $2 \times 1$ reconstructions, respectively. 
a)

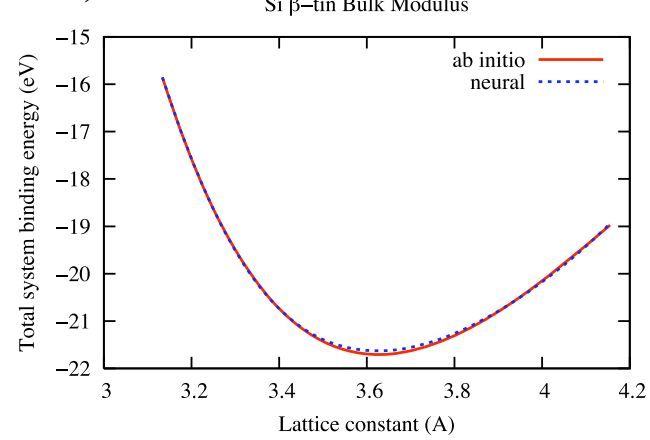

c)

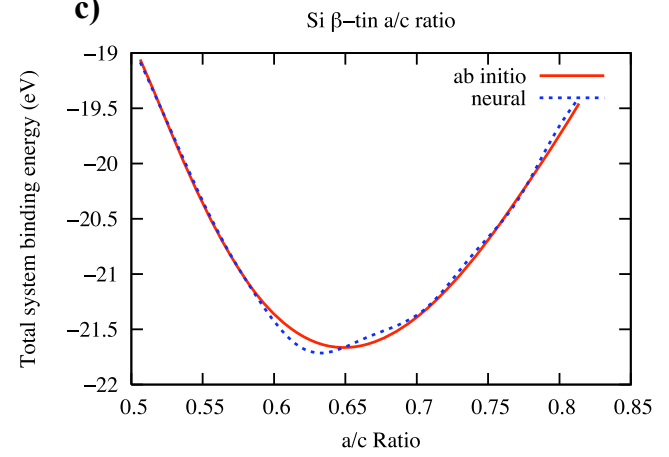

b)

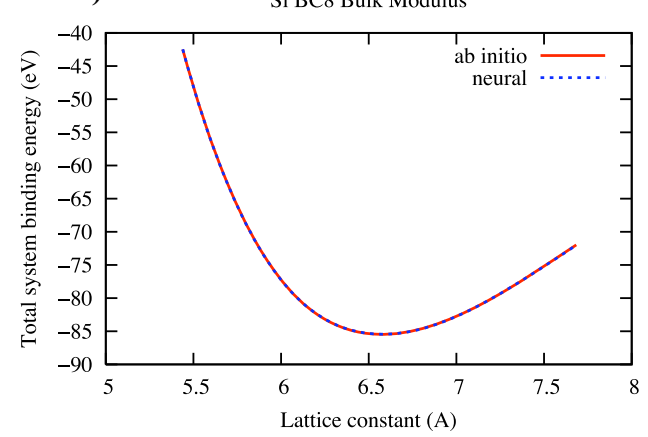

d)

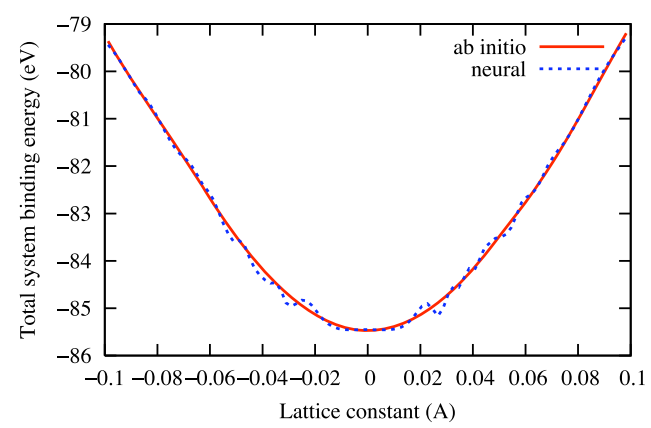

FIG. 8: Neural network fits for silicon $\beta$-tin and BC8 lattices: (a) and (b) hydrostatic compression, (c) $\beta$-tin $a / c$ ratio, and (d) $\mathrm{BC} 8 C^{\prime}$ distortion curves. 

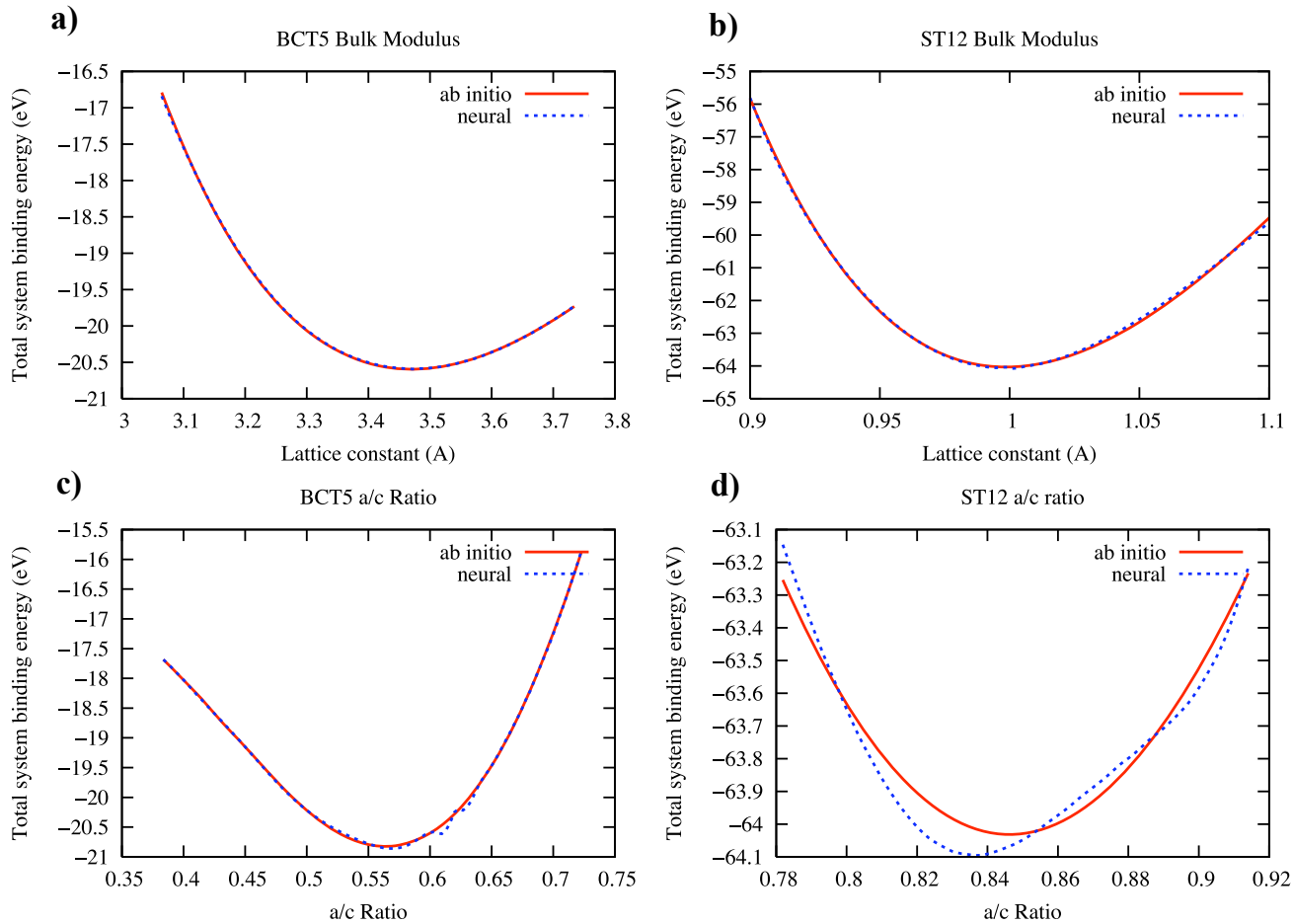

FIG. 9: Neural network fits for silicon ST12 and BCT5 lattices: (a) and (b) hydrostatic compression, (c) and (d) $a / c$ ratios. 
APPENDIX: NEURAL NETWORK PARAMETERS

\section{Biases}

\begin{tabular}{|l|r|r|r|r|}
\hline & Layer 1 & Layer 2 & Layer 3 & Layer 4 \\
\hline$b_{1}$ & -24.19488757 & -2.453917947 & -1.731891107 & 235.8214374 \\
$b_{2}$ & 7.855650659 & -2.666861068 & -83.91499543 & \\
$b_{3}$ & -28.20953658 & 0.663331165 & -5.506684641 & \\
$b_{4}$ & -6.000666175 & 1.270167597 & -2.917664551 & \\
$b_{5}$ & 1.355351589 & -1.743372541 & 14.58172457 & \\
$b_{6}$ & $-4.93 \mathrm{E}-02$ & 0.115779703 & 30.86442975 & \\
$b_{7}$ & 65.30609095 & 0.984555389 & -12.38780015 & \\
$b_{8}$ & 13.87230391 & -1.008096389 & -119.0126487 & \\
$b_{9}$ & 14.60655681 & 0.25026836 & -38.05256282 & \\
$b_{10}$ & 15.71305935 & 0.346107045 & -6.666571419 & \\
$b_{11}$ & 24.43823784 & -0.215688631 & 4.40808851 & \\
$b_{12}$ & -22.08179355 & 0.818524672 & -17.27846627 & \\
$b_{13}$ & -4.677000448 & -0.419191786 & 21.41614412 & \\
\hline
\end{tabular}




\section{Weights A}

\begin{tabular}{|c|c|c|c|c|c|}
\hline$w_{i j}^{A}$ & \multicolumn{5}{|c|}{$i=$} \\
\hline$j=$ & 1 & 2 & 3 & 4 & 5 \\
\hline 1 & 9.461963084 & -20.44077585 & -19.63391137 & -12.61604489 & 7.790530219 \\
\hline 2 & -6.476773757 & 1.00927534 & -22.77740546 & -2.283720521 & -4.905044473 \\
\hline 3 & 15.35978591 & -8.314029502 & 24.87778028 & 3.111485278 & -17.49787411 \\
\hline 4 & -1.845622721 & -4.540684498 & 4.98740141 & 0.179084083 & -3.956729862 \\
\hline 5 & 0.483804234 & -6.713045382 & 7.035476613 & 0.820614138 & 12.31156997 \\
\hline 6 & 18.40694178 & -3.454207762 & 17.0551604 & 4.70125412 & -10.40131828 \\
\hline 7 & 4.622497864 & 12.60348526 & -21.22083072 & 7.443793936 & -12.33292849 \\
\hline 8 & -4.517640107 & -7.258905479 & -25.97994224 & -4.566760786 & -0.35921081 \\
\hline 9 & 3.0619702 & -3.757521144 & 3.090223106 & -4.128690031 & 7.07664859 \\
\hline 10 & 39.91185325 & 3.579947725 & 56.63097537 & 4.514400644 & 27.17420149 \\
\hline 11 & 0.425452957 & -13.75460726 & -32.76709282 & -5.658170093 & -20.97524441 \\
\hline 12 & -17.98557669 & 24.82949678 & 28.1940987 & 8.363833038 & 0.531683196 \\
\hline 13 & -56.46301859 & 3.806683698 & -29.54367051 & 4.670613522 & -12.60586624 \\
\hline$w_{i j}^{A}$ & \multicolumn{5}{|c|}{$i=$} \\
\hline$j=$ & 6 & 7 & 8 & 9 & 10 \\
\hline 1 & -3.395954035 & 81.18994778 & -21.35032885 & 22.94784455 & 1.78008499 \\
\hline 2 & 0.650159733 & 84.2423039 & 1.418745777 & 2.23318286 & 0.834558921 \\
\hline 3 & 14.67310831 & -186.5552164 & -8.615825601 & -2.665803274 & -38.51779356 \\
\hline 4 & 14.67856035 & -15.71486121 & -0.957659426 & -4.400316504 & -3.932550473 \\
\hline 5 & -1.708907626 & -47.17698904 & 1.455487292 & -9.039747044 & -10.42895637 \\
\hline 6 & $7.59 \mathrm{E}-02$ & 18.02323591 & -17.72685553 & 3.583088661 & -0.882705823 \\
\hline 7 & 7.521569215 & 9.397421886 & 6.119598278 & -9.871760612 & 12.83699794 \\
\hline 8 & 13.6157047 & -13.42783713 & -5.180494846 & 4.602055195 & -2.419326148 \\
\hline 9 & 0.587780948 & -1.911801507 & 4.757399246 & -7.134793967 & -20.60146823 \\
\hline 10 & -31.44429051 & -2.974290699 & 4.645659378 & -2.834087738 & -7.453378403 \\
\hline 11 & 19.36500956 & -14.34285415 & -13.08481031 & 6.245148008 & 2.152761505 \\
\hline 12 & -18.04270521 & 7.886091286 & 6.986650979 & -10.4991478 & -0.105569345 \\
\hline 13 & 25.25026 & -34.48128615 & 20.22731503 & -8.975026859 & -0.822776451 \\
\hline
\end{tabular}




\begin{tabular}{|l|r|r|r|}
\hline$w_{i j}^{A}$ & \multicolumn{2}{|c|}{$i=$} \\
$j=$ & 11 & 12 & 13 \\
\hline 1 & 2.688011573 & -17.44369395 & -4.262926602 \\
2 & -19.86539355 & -3.759714138 & 2.66931686 \\
3 & 0.951869084 & -17.14761305 & 12.66382983 \\
4 & 5.046154211 & -10.21429013 & -7.081107604 \\
5 & -13.86771443 & 13.89652985 & 4.196947351 \\
6 & -14.1782213 & 26.16974513 & 7.017171559 \\
7 & -15.43387403 & -24.83977332 & 10.62038776 \\
8 & -12.54879186 & -12.40758633 & 12.76277702 \\
9 & -5.412492575 & -30.78469433 & -1.405575552 \\
10 & 13.36817728 & 63.07284615 & -14.00848288 \\
11 & -27.1341802 & -41.95219027 & -4.688905298 \\
12 & 13.15358259 & 45.66191043 & 4.882555594 \\
13 & 49.90545105 & -12.62130376 & 12.61275442 \\
\hline
\end{tabular}




\section{Weights C}

\begin{tabular}{|c|c|c|c|c|c|}
\hline$w_{i j}^{C}$ & \multicolumn{5}{|c|}{$i=$} \\
\hline$j=$ & 1 & 2 & 3 & 4 & 5 \\
\hline 1 & -1.202023323 & -35.52519425 & -11.45782068 & -2.241729699 & 15.82819316 \\
\hline 2 & -1.155470927 & -94.564796 & 0.199819673 & -4.920619951 & 49.38988853 \\
\hline 3 & -1.584969915 & 113.3058827 & -2.918013493 & -4.774123161 & -166.8331578 \\
\hline 4 & -0.893163571 & 169.4628138 & 3.414866597 & 8.459631626 & -54.59454296 \\
\hline 5 & -0.619151556 & 11.7345258 & 1.394553633 & 1.793400921 & 58.3989406 \\
\hline 6 & 0.473042635 & 52.98677948 & 1.898383384 & 2.348990355 & 14.67358348 \\
\hline 7 & -0.363095154 & -51.56466702 & 1.198738757 & 0.656264325 & 74.83230022 \\
\hline 8 & -0.944699692 & -79.512631 & 0.891859259 & 3.217610689 & 117.3601142 \\
\hline 9 & -0.37081023 & 48.09674074 & 0.754604515 & 1.050526286 & 121.235384 \\
\hline 10 & -5.028041395 & -271.2684307 & -12.7981033 & -19.58010818 & 18.30125128 \\
\hline 11 & -1.1128842 & -14.61297761 & -3.360990006 & -9.473884072 & -19.17167298 \\
\hline 12 & $-4.75 \mathrm{E}-02$ & 49.91715288 & 5.751664225 & 6.07655642 & -27.88600929 \\
\hline 13 & -2.338650262 & 25.05832149 & -9.956172095 & -20.10100426 & -112.1362461 \\
\hline$w_{i j}^{C}$ & \multicolumn{5}{|c|}{$i=$} \\
\hline $\mathrm{j}=$ & 6 & 7 & 8 & 9 & 10 \\
\hline 1 & -34.66069943 & -80.22811538 & -102.6083295 & 26.68391154 & -2.898591684 \\
\hline 2 & -33.47530527 & -24.14737588 & 18.33914464 & -9.284152879 & 8.246078896 \\
\hline 3 & -43.99085672 & 56.11579445 & -138.1436843 & -16.13415365 & 7.771869081 \\
\hline 4 & 1.634132811 & -63.2819568 & 112.9569836 & 46.67488873 & -65.09005738 \\
\hline 5 & 110.4634758 & -24.49421405 & 93.33184254 & 15.21434978 & -47.50476792 \\
\hline 6 & 18.47781052 & 122.4442017 & -26.88090731 & -34.26678485 & 37.21018187 \\
\hline 7 & 10.21157666 & -33.45781345 & 98.50625491 & 15.10191019 & -13.03714103 \\
\hline 8 & 22.58951838 & 36.20763857 & 103.2831947 & -18.65054613 & 23.22989585 \\
\hline 9 & -32.25200602 & -28.46729992 & 110.2847001 & 13.32999943 & -32.11564924 \\
\hline 10 & 89.7508315 & 95.58481201 & 50.1862502 & 25.80852372 & 56.03674822 \\
\hline 11 & 12.86929136 & -37.352155 & -42.0859243 & -20.97220663 & 44.21265407 \\
\hline 12 & -47.51164914 & -7.364238907 & -47.42658648 & 23.30137444 & -7.038400094 \\
\hline 13 & -75.38445241 & -61.17040337 & -79.5974037 & -10.87281086 & 16.86505173 \\
\hline
\end{tabular}




\begin{tabular}{|l|r|r|r|}
\hline$w_{i j}^{C}$ & \multicolumn{3}{|c|}{$i=$} \\
$j=$ & 11 & 12 & 13 \\
\hline 1 & 33.70972554 & -55.18276164 & -39.26102557 \\
2 & -15.57227185 & -48.05974716 & 20.94630083 \\
3 & -45.70629919 & 128.6421196 & 46.54543631 \\
4 & 22.15244641 & 14.96256614 & -24.99682686 \\
5 & -62.68588172 & 18.74586001 & 64.94263442 \\
6 & -69.54688373 & -26.51129092 & 74.09407719 \\
7 & 19.20190294 & -27.11455564 & -21.0840884 \\
8 & -2.059265461 & 84.18058581 & 2.501332706 \\
9 & $1.94 \mathrm{E}-03$ & 124.1321055 & 4.362968182 \\
10 & 20.23036403 & -40.10783017 & -74.45136289 \\
11 & 47.35335092 & -101.4537433 & -47.73902303 \\
12 & -22.76828654 & -108.4519552 & 24.2397533 \\
13 & 48.19670458 & 5.177957459 & -46.32321764 \\
\hline
\end{tabular}

\section{Weights D}

\begin{tabular}{|l|r|}
\hline Weight & Value \\
\hline$w_{1,1}^{D}$ & -1.581981887 \\
$w_{1,2}^{D}$ & -81.1723725 \\
$w_{1,3}^{D}$ & -11.25538492 \\
$w_{1,4}^{D}$ & -20.83565831 \\
$w_{1,5}^{D}$ & -73.53671911 \\
$w_{1,6}^{D}$ & -3.092725528 \\
$w_{1,7}^{D}$ & -9.683353238 \\
$w_{1,8}^{D}$ & -47.07268377 \\
$w_{1,9}^{D}$ & -104.4083203 \\
$w_{1,10}^{D}$ & -89.93911101 \\
$w_{1,11}^{D}$ & -55.57487894 \\
$w_{1,12}^{D}$ & -6.479432706 \\
$w_{1,13}^{D}$ & -53.40840723 \\
\hline
\end{tabular}




\section{REFERENCES}

[1] F. Ercolessi, M. Parrinello, and E. Tosatti, Philos. Mag. A 58 (1988).

[2] M. S. Daw and M. I. Baskes, Phys. Rev. B 29 (1984).

[3] S. M. Foiles, M. I. Baskes, and M. S. Daw, Phys. Rev. B 33 (1986).

[4] M. Finnis and J. Sinclair, Philos. Mag. A 50 (1984).

[5] F. Stillinger and T. A. Weber, Phys. Rev. B 31 (1985).

[6] J. Tersoff, Phys. Rev. B 37 (1988).

[7] R. Car and M. Parrinello, Phys. Rev. Lett. 55 (1985).

[8] L. Verlet, Phys. Rev. 159 (1967).

[9] E. González-Romera, M. Ángel Jaramillo-Morán, and D. Carmona-Fernández, Comput. Ind. Eng. 52, 336 (2007).

[10] H. Bhadeshia, ISIJ Int. 39, 966 (1999).

[11] E. Metzbower, J. deLoach, S. Lalam, and H. Bhadeshia, Sci. Tech. Weld. Join. 6, 116 (2001).

[12] X. Shi, L. Wang, N. Kariuki, C. Zhong, and S. Lu, Sens. and Act. B 117, 65 (2006).

[13] A. Bholoa, S. D. Kenny, and R. Smith, Nucl. Inst. Meth. Phys. Res. B 255 (2007).

[14] J. Behler and M. Parrinello, Phys. Rev. Lett. 98 (2007).

[15] M. Baskes, Mater. Chem. Phys. 50 (1997).

[16] R. F. W. Bader, Atoms in Molecules: A Quantum Theory (Clarendon Press, Oxford, 1994).

[17] E. Sanville, S. D. Kenny, R. Smith, and G. Henkelman, J. Comp. Chem. 28 (2007). 